\title{
Lipoprotein(a): a not-so-well-known risk factor for the development of cardiovascular disease in patients with type 2 diabetes mellitus
}

Min Kyong Moon

Department of Internal Medicine, Seoul Metropolitan Government Seoul National University Boramae Medical Center, Seoul, Korea

Received: October 24, 2016 Accepted: October 26, 2016

\section{Correspondence to}

\section{Min Kyong Moon, M.D.}

Department of Internal Medicine, Seoul Metropolitan Government Seoul National University Boramae Medical Center, 20 Boramae-ro 5-gil, Dongjak-gu, Seoul 07061, Korea

Tel: +82-2-870-2226

Fax: +82-2-831-0714

E-mail:mkmoon@snu.ac.kr

\section{See Article on Page 1110-1119}

Lipoprotein(a) (Lp(a)), first discovered by Kare Berg in 1963, is an independent risk factor for the development of atherosclerotic cardiovascular disease (CVD) [1]. The structure of $L p(a)$ is similar to that of low density lipoprotein; both complexes contain a lipid core surrounded by apolipoprotein B (apoB), but in $\mathrm{Lp}(\mathrm{a})$, a unique apolipoprotein, apolipoprotein A (apo(a)), is covalently bound to apoB via a disulfide bond [1]. Apo(a) shares high sequence homology with plasminogen but has no fibrinolytic activity. Rather, apo(a) enhances coagulation and compromises clot lysis [2]. The plasma concentrations of $\mathrm{Lp}(\mathrm{a})$ are (principally) determined genetically and differ according to ethnicity [3]. Caucasians have lower median plasma levels than do Africans, and Chinese and Asian populations have lower levels than do Caucasians [4,5].

In this issue of the Korean Journal of Internal Medicine, Lim et al. [6] report that an elevated Lp(a) level is an independent predictor of the development of CVD in Korean patients with type 2 diabetes mellitus (T2DM). This has high importance, as very limited information is available on the range of $\mathrm{Lp}(\mathrm{a})$ levels and the association of $\mathrm{Lp}(\mathrm{a})$ with CVD in Koreans. The study is particularly meaningful because the cited authors prospectively followed up a relatively large patient cohort for over 10 years. New CVDs developed in $24.2 \%$ of patients during the 11.1 years of follow-up. This confirms that patients with $\mathrm{T} 2 \mathrm{DM}$ are at a very high risk of CVD. However, the incidence of CVD in the cited study seems to be much higher than those in other recent studies. This may be because all patients were enrolled in 2003 to 2004 , the proportion treated with statins was only $11.6 \%$, and the baseline mean glycated hemoglobin (HbArc) level was relatively high (8.9\%). CVD was much more prevalent among patients with mean HbAic levels $>9.0 \%$ than among those with mean HbAic levels < 7.0\% over the study period. The work confirms that strict glycemic control can reduce the CVD risk in patients with $\mathrm{T} 2 \mathrm{DM}$ of relatively long duration.

A high Lp(a) level increases the CVD risk, particularly that in high-risk groups. However, it is very difficult to determine the $\mathrm{Lp}(\mathrm{a})$ level indicative of risk. Some studies use 30 or $50 \mathrm{mg} / \mathrm{dL}$ as the cutoff value; others define highrisk patients as those in the highest quartile or quintile. Lp(a) plasma concentrations differ according to ethnicity [3]; thus, more research is required 
to determine the level associated with CVD risk in Koreans. In a cross-sectional survey of 14,516 apparently healthy Koreans, the 25th percentile, median, and 75th percentile Lp(a) levels were 9.4, 13.2, and $23.8 \mathrm{mg} / \mathrm{dL}$, respectively [7]. These levels were similar to those in the study by Lim et al. [6], of 8.3, 16.7, and $35.1 \mathrm{mg} / \mathrm{dL}$, respectively, in Korean patients with T2DM. The hazard ratio for CVD development was 2.37 for the 4 th versus the ist $\mathrm{Lp}(\mathrm{a})$ quartile, and the median $\mathrm{Lp}(\mathrm{a})$ level in the 4th quartile was $55.7 \mathrm{mg} / \mathrm{dL}$ (range, 43.1 to 75.3 ).

Generally, Lp(a) levels are not elevated in patients with T2DM compared with nondiabetic subjects. Furthermore, in an earlier report [8], Lp(a) levels were not associated with the duration of diabetes or the extent of hyperglycemia. In another Chinese study, however, serum $\operatorname{Lp}(\mathrm{a})$ concentrations were inversely associated with T2DM, prediabetes, and insulin resistance [9]. The question of whether Lp(a) levels differ between males and females remains controversial. Some prospective studies found no significant sex-related difference in $\mathrm{Lp}(\mathrm{a})$ concentrations $[10,11]$. However, in other studies, including that by Lim et al. [6], Lp(a) levels in females were higher than those in males $[6,12,13]$. In the study by Lim et al. [6], CVD developed more often in females. Therefore, the elevated CVD risk may be associated with the higher Lp(a) levels in females, although other risk factors, including age, need to be compared between males and females.

Plasma concentrations of $\mathrm{Lp}(\mathrm{a})$ are (principally) determined genetically; lifestyle modifications (diet and exercise) do not reduce these levels. Statins do not affect $\mathrm{Lp}(\mathrm{a})$ levels, but niacin reduces the levels by $20 \%$ to $30 \%[2]$. However, the Atherothrombosis Intervention in Metabolic Syndrome with Low HDL/High Triglycerides: Impact on Global Health Outcomes Trial showed that addition of niacin to statin therapy did not further reduce the number of CVD events [14]. It is thus uncertain whether $\mathrm{Lp}(\mathrm{a})$-reduction therapy can reduce the CVD risk. The Lp(a) level may be pathogenetically associated with CVD, but at present, that level is of limited clinical application when used to stratify risk. Intra-individual long-term variations in $\mathrm{Lp}$ (a) levels are very low in the absence of specific treatments; a single determination is usually sufficient to determine the general risk. Some drugs under clinical development have been shown to decrease Lp(a) levels; these include mi- pomersen, proprotein convertase subtilisin/kexin type 9 ( PCSK $\left._{9}\right)$ inhibitors, cholesteryl ester transfer protein (CETP) inhibitors, and eprotirome. The apoB synthesis inhibitor mipomersen decreased Lp(a) concentrations by approximately 30\% [2]. Anacetrapib, a CETP inhibitor $[1,2]$ and the thyroid hormone analog eprotirome [2] each decreased $\mathrm{Lp}(\mathrm{a})$ levels by approximately $40 \%$. The CVD outcomes of patients taking such drugs would yield valuable information on whether $\mathrm{Lp}(\mathrm{a})$-reduction treatment reduces the incidence of CVD events. Fibrate derivatives increase plasma $L p(a)$ concentrations in subjects with hypertriglycemia, and changes in such levels were negatively correlated with changes in triglyceride concentrations [15]. This may explain why fibrate exerts a relatively low CVD preventative effect compared with statins. Apart from CVD, the Lp(a) level was also reported to be associated with diabetic retinopathy and nephropathy in patients with T2DM.

In conclusion, the plasma Lp(a) level may be a useful risk factor for the development of CVD in Korean patients with T2DM. However, no clear cutoff value indicative of CVD risk in Koreans has yet been defined. Indeed, even a range of values is lacking. More research is required. Presently, Lp(a) screening can be used only to stratify those at risk of CVD. However, randomized controlled trials exploring whether selective reduction of $\mathrm{Lp}$ (a) levels improves cardiovascular outcomes may alter this view in the future.

\section{Conflict of interest}

No potential conflict of interest relevant to this article was reported.

\section{REFERENCES}

1. Hoover-Plow J, Huang M. Lipoprotein(a) metabolism: potential sites for therapeutic targets. Metabolism 2013;62:479491.

2. Berthold HK, Gouni-Berthold I. Hyperlipoproteinemia(a): clinical significance and treatment options. Atheroscler Suppl 2013;14:1-5.

3. Nordestgaard BG, Langsted A. Lipoprotein(a) as a cause of cardiovascular disease: insights from epidemiology, genetics, and biology. J Lipid Res 2016 Sep 27 [Epub]. http:// doi.org/10.1194/j1r.Ro71233. 
4. Sandholzer C, Hallman DM, Saha N, et al. Effects of the apolipoprotein(a) size polymorphism on the lipoprotein(a) concentration in 7 ethnic groups. Hum Genet 1991;86:607-614.

5. Guan W, Cao J, Steffen BT, et al. Race is a key variable in assigning lipoprotein(a) cutoff values for coronary heart disease risk assessment: the Multi-Ethnic Study of Atherosclerosis. Arterioscler Thromb Vasc Biol 2015;35:9961001.

6. Lim TS, Yun JS, Cha SA, et al. Elevated lipoprotein(a) levels predict cardiovascular disease in type 2 diabetes mellitus: a 10-year prospective cohort study. Korean J Intern Med 2016;31:1110-1119.

7. Lee HJ, Pae JC, Sung KC, et al. Distribution of serum lipoprotein(a) level and its association with other risk factors in apparently healthy Korean. Korean Circ J 2006;36:150158.

8. Haffner SM, Morales PA, Stern MP, Gruber MK. Lp(a) concentrations in NIDDM. Diabetes 1992;41:1267-1272.

9. Ding L, Song A, Dai M, et al. Serum lipoprotein (a) concentrations are inversely associated with $\mathrm{T} 2 \mathrm{D}$, prediabetes, and insulin resistance in a middle-aged and elderly Chinese population. J Lipid Res 2015;56:920-926.

10. Jenner JL, Ordovas JM, Lamon-Fava S, et al. Effects of age, sex, and menopausal status on plasma lipoprotein(a) levels: the Framingham Offspring Study. Circulation 1993;87:1135-1141.

11. Marcovina SM, Albers JJ, Jacobs DR Jr, et al. Lipoprotein[a] concentrations and apolipoprotein[a] phenotypes in Caucasians and African Americans: the CARDIA study. Arterioscler Thromb 1993;13:1037-1045.

12. Emerging Risk Factors Collaboration, Erqou S, Kaptoge $\mathrm{S}$, et al. Lipoprotein(a) concentration and the risk of coronary heart disease, stroke, and nonvascular mortality. JAMA 2009;302:412-423.

13. Brown SA, Hutchinson R, Morrisett J, et al. Plasma lipid, lipoprotein cholesterol, and apoprotein distributions in selected US communities: the Atherosclerosis Risk in Communities (ARIC) Study. Arterioscler Thromb 1993;13:11391158.

14. AIM-HIGH Investigators, Boden WE, Probstfield JL, et al. Niacin in patients with low HDL cholesterol levels receiving intensive statin therapy. N Engl J Med 2011;365:22552267.

15. Ko HS, Kim CJ, Ryu WS. Effect of fenofibrate on lipoprotein(a) in hypertriglyceridemic patients: impact of change in triglyceride level and liver function. J Cardiovasc Pharmacol 2005;46:405-411. 